\title{
ACETALDEHYDE SUPPRESSES GROWTH, CHANGES CONIDIA MORPHOLOGY AND REDUCES THE PRODUCTION OF ADENOSINE 3',5'-CYCLIC MONOPHOSPHATE IN A DOSE DEPENDENT MANNER IN ALTERNARIA ALTERNATA SHORT COMMUNICATION
}

\author{
Mivuyo S. Mbovane, ${ }^{1}$ Venkata S. R. GangiReddygari, ${ }^{1}$ \\ HLENGILIZWE NyONI ${ }^{2}$ and KhAYALETHU NTUSHELO ${ }^{1}$ * \\ ${ }^{1}$ Department of Agriculture and Animal Health, University of South Africa, Florida, 1710 South Africa \\ ${ }^{2}$ Nanotechnology and Water Sustainability Research Unit, University of South Africa, \\ Florida, 1710 South Africa
}

(Received: May 22, 2017; accepted: August 1, 2017)

\begin{abstract}
One-day-old cultures of the plant pathogenic fungus Alternaria alternata were exposed to $0 \%, 5 \%$ and $10 \%$ acetaldehyde mixed with distilled water. Fungal growth data showed that, overall, the $5 \%$ and the $10 \%$ acetaldehyde treatments significantly inhibited the growth of $A$. alternata, and that acetyldehyde also facilitated maturity and multicellularity of fungal conidia. The increase of the acetyldehyde dose also caused correlated decrease of adenosine 3',5'-cyclic monophosphate produced by A. alternata.
\end{abstract}

Keywords: Acetaldehyde - adenosine 3',5'-cyclic monophosphate - Altenaria alternate - growth conidia

The phytopathogenic fungus Alternaria alternata (Fr.) Kreissler can be controlled with fumigants $[4,8]$ like acetaldehyde. Acetaldehyde limits decay caused by A. alternata in grapes [3], reduces its colony growth in Petri dishes [6] as well as inhibit spore germination and development of $A$. alternata [1]. This study represents the first report on the combined effect of acetaldehyde on growth, conidial morphology and production of adenosine 3',5'-cyclic monophosphate in A. alternata.

Fresh $3 \mathrm{~mm}$ PDA mycelia plugs of $A$. alternata (strain PPRI 12409) were placed mycelia down on carrot agar media in Petri dishes and were cultured for 24 hours at $28{ }^{\circ} \mathrm{C}$. After the 24 -hour incubation, $0 \%, 5 \%$ and $10 \%$ distilled water acetaldehyde solutions (poured in Eppendorf caps) were enclosed (two Eppendorf caps per Petri dish) with the fungi, and incubation continued for an additional 24 hours for one set of cultures, for 48 hours for another set, 96 hours and 120 hours for additional sets. The Petri dishes were sealed with Parafilm throughout the incubation period. The experiment was conducted in triplicate and in total 36 plates were examined. At each 24-hour interval, fungal colony diameter was measured and the fungal culture was observed under the XB 43 Olympus $^{\circledR}$ light microscope $(40 \times)$. Fungal growth data

\footnotetext{
*Corresponding author; e-mail address: ntushk@unisa.ac.za
} 


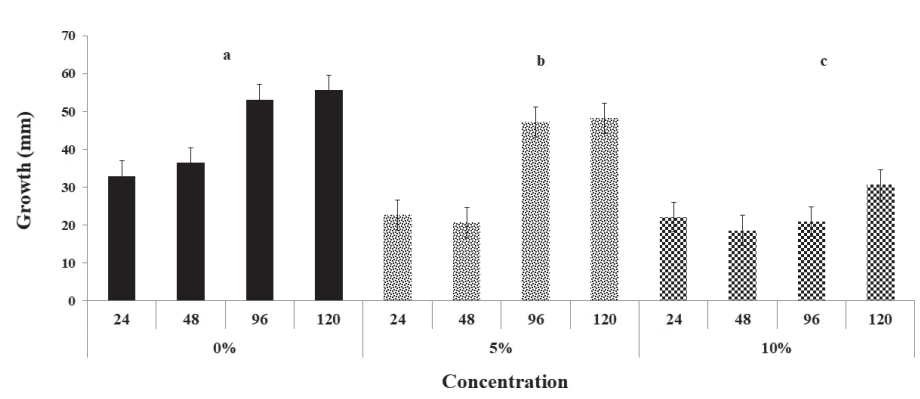

Fig. 1. Growth of Altenaria alternata after exposure to different concentrations $(0 \%, 5 \%$ and $10 \%)$ of acetaldehyde observed at 24, 48, 96 and 120 hours after exposure. The letters ( $a, b$ and c) over the bars indicate overall statistical significant differences $(p=0.05)$ between the treatments (percentage acetaldehyde) (three observations per treatment $n=3$ ). Generally, growth tapered from 96 hours to 120 hours after exposure to acetaldehyde

(mycelial colony diameters) was analysed by analysis of variance using Statistica. Furthermore, the 24-hour cultures (most chemical response expected) were analysed using the LCMS-8040 triple quadrupole mass spectrometer (Shimadzu).

Overall, growth of cultures exposed to $0 \%, 5 \%$ and $10 \%$ acetaldehyde differed significantly (observation and statistical analysis) with each other (Fig. 1). Results obtained from this work showed that exposure of A. alternata to 5\% and $10 \%$ acetaldehyde inhibits the growth of this fungus from as early as 24 hours after exposure. This was, however, not a new phenomenon as this had already been found by Almenar et al. [1]. Our interest was in the effect of acetaldehyde not only on the growth and morphology but also on chemical responses of A. alternata. After 48 hours of incubation with acetaldehyde, conidia of A. alternata treated with $5 \%$ and $10 \%$ acetaldehyde showed multiple septation, as compared to the control $(0 \%)$ treatment whose conidia were rounded mostly with no septation (Fig. 2). Similarly, Avissar et al. [2] observed inhibition of germ tube elongation (because of amino acid and sugar leakage from the mycelium) in Rhizopus stolonifer and Botrytis cinerea after exposure to acetaldehyde. LCMS-8040 triple quadrupole mass spectrometer (Shimadzu) analysis of 24-hour cultures showed that the production of adenosine 3',5'-cyclic monophosphate (cAMP) had been suppressed in a dose-dependent manner. The LCMS-8040 triple quadrupole mass spectrometer chromatogram peak area (representing the quantity of cAMP) was 3661 for the $0 \%$ acetaldehyde treatment,

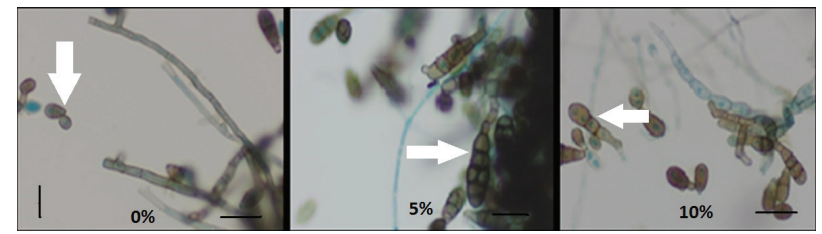

Fig. 2. Microscope images showing the altering of morphogenesis caused by exposure of Alternaria alternata to acetaldehyde. The spores become multicellular with increasing acetaldehyde treatment at 48 hours after exposure. See white arrows pointing at the spores. The scale bar represents $20 \mu \mathrm{m}$ 
was halved to 1717 by the $5 \%$ acetaldehyde treatment and no cAMP was detected in the $10 \%$ acetaldehyde treatment. Similarly, Scott and Solomon [10] also found that treatment of Neurospora crassa with atropine and antimalarials resulted in decreased fungal growth as well as in a reduction of cAMP, confirming the findings of Scott et al. [9] that cAMP influences growth and shape in N. crassa and that cAMP is implicated in the differentiation of many fungi [5]. Moreover, dimorphic behaviour has often been linked to intracellular levels of this cyclic nucleotide [7]. These results are, however, preliminary and a detailed investigation on the influence of acetaldehyde on the growth, conidial morphology and chemical composition of A. alternata still requires extensive investigation.

\section{ACKNOWLEDGMENTS}

The Agricultural Research Council of South Africa provided the fungi and Dr Sheku Alfred Kanu analysed the fungal growth data.

\section{REFERENCES}

1. Almenar, E., Auras, R., Wharton, P., Rubino, M., Bruce, H. (2007) Release of acetaldehyde from $\beta$-cyclodextrins inhibits postharvest decay fungi in vitro. J. Agric. Food Chem. 55, 7205-7212.

2. Avissar, I., Droby, S., Pesis, E. (1990) Characterization of acetaldehyde effects on Rhizopus stolonifer and Botrytis cinerea. Ann. Appl. Biol. 116, 213-220.

3. Avissar, I., Pesis, E. (1991) The control of postharvest decay in table grapes using acetaldehyde vapours. Ann. Appl. Biol. 118, 229-237.

4. Feng, W., Chen, J., Zheng, X., Liu, Q. (2011) Thyme oil to control Alternaria alternata in vitro and in vivo as fumigant and contact treatments. Food Control 22, 78-81.

5. Pall, M. L. (1981) Adenosine 3':5'-monophosphate in fungi. Microbiol. Rev. 45, 462-491.

6. Prusky, D., Perez, A., Zutkhi, Y., Ben-Arie, R. (1997) Effect of modified atmosphere for control of black spot, caused by Alternaria alternata, on stored Persimmon fruits. Phytopathology 87, 203-208.

7. San-Blas, G., San-Blas, F. (1984) Molecular aspects of fungal dimorphism. CRC Crit. Rev. Microbiol. $11,101-127$.

8. Song, J., Hildebrand, P. D., Fan, L., Forney, C. F., Renderos, W. E., Campbell-Palmer, L., Doucette, C. (2007) Effect of hexanal vapor on the growth of postharvest pathogens and fruit decay. J. Food Sci. $72,108-112$

9. Scott, W. A., Mishra, N. C., Tatum, E. L. (1973) Biochemical genetics of morphogenesis in Neurospora. Brookhaven. Symp. Biol. 25, 1-18.

10. Scott, W. A., Solomon, B. (1975) Adenosine 3',5'-cyclic monophosphate and morphology in Neurospora crassa: drug-induced alterations. J. Bacteriol. 122, 454-463. 